\title{
RADIATION HAZARDS
}

\author{
Katherine Williams, B.Sc., M.B., B.Ch., M.R.C.P., D.I.H. \\ formerly Principal Medical Officer, U.K.A.E.A. \\ Division Head, Medical Division, Atomic Energy Research Establishment, Harwell
}

THE nuclear age was born in December 1942 when the first chain-reacting pile was found to work. Radiation had been known since Roentgen's discovery of the X-ray and Becquerel's of radioactivity. During the early years of this century, knowledge of the dangers of radiation was acquired by painful experience. Radiation sources, at that time, were confined to relatively small amounts of radium and other naturally occurring radioactive substances and to X-ray tubes. Although these radiation sources were the products of the new discoveries, mankind has always been exposed to radiation. The background, to which we are all exposed throughout our lifetime, comes from cosmic rays reaching us from interstellar space, gamma rays from naturally occurring radioactive substances in rocks and soils, atmospheric radon, and also internal radiation from the radioactive constituents of the body such as the radioactive isotope potassium-40. Rocks, shale and soil contain thorium and uranium and their daughter products in varying quantities, and from them, there is leaching by rain into rivers and streams and uptake by plants. Thus very minute, but measurable, amounts are found in food and in drinking water, and from this intake, radium gradually builds up in the human body until it reaches about $10^{-4}$ microcuries of radium in the adult. In assessing the hazards of radiation in the range of very low dosage, the radiation from natural sources is taken as the "yardstick" for comparison. From these sources of radiation, the average person receives about $60 \mathrm{mrad}$ of radiation exposure per year.

The discovery of nuclear fission and the subsequent building of nuclear reactors has enormously widened the scope and increased the quantity of radionuclides available for research and therapy in medicine and use in industry.

The proliferation of machines such as the synchrotron and linear accelerators has greatly widened the scope and increased the intensity of radiations available for research and therapy in medicine and use in industry.

Some of the new man-made radioactive substances were unknown previously and have no physiological analogue. In the nuclear fission of uranium, the nucleus splits into two approximately equal parts creating elements about half the mass of the uranium atom. Some of these radionuclides are very short- lived and it is customary to consider chiefly those that are present in the fission product mixture after a decay period of one week. Members of the alkaline earths and the lanthanide rare earths are produced in high yield, and isotopes of strontium barium, cesium, lanthanum, cerium, zirconiumg cobalt, ruthenium, tellurium and zinc account fore. almost all the total radioactivity. In addition, in at nuclear reactor, really heavy elements, heavier thain uranium-238 are made from it - the trans-uranice elements. Plutonium is produced in quantitie which suggest that it will be used as a reactor fuet for future reactors. On this account, it is importanto As it is not found in nature (except in very minute quantities) it was previously quite unknown. Radio strontium, one of the fission products, is an im $\overrightarrow{0}$ portant nuclide, since it is produced in high yield, i\$ relatively long-lived and is a bone-seeking element: when taken into the body. Plutonium is also sto in the skeleton after intake. The toxicity of thesse substances can be inferred from comparison with radium, for which there is considerable experiences and knowledge.

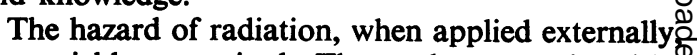
was quickly recognised. The erythema produced bye $\mathrm{X}$-rays was noted within a few days after the dis $\overrightarrow{{ }_{0}}$ covery of this radiation and, shortly after the discovery of radium, similar damage to the skin? was found. Thirty years were to elapse before the severe lesions caused by the internal deposition of radium in the body were recognised and described $?$. This latent period is important in industrial medica practice.

Outside the body, radioactive substances which? emit gamma rays are the most dangerous because they are penetrating, while alpha and beta emitters have low penetrating power. Inside the body, the order of importance is reversed. Alpha and beta․ particles have a short path and so their energy is aln absorbed in a small volume of tissue near the radiat ing source, and cells in proximity may receive lethal amount of radiation or the damage may bow such that subsequently they undergo malignan change.

In the case of external exposure to radiation, it is relatively easy to measure and to estimate the dose to a given organ or tissue. When exposure to an external source has occurred and been recognised $\stackrel{0}{\mathbb{D}}$ it can be terminated fairly quickly, and in general 
an external radiation field that is hazardous is relatively easy to detect and monitor.

For internal exposure, preventive measures are exceedingly important because of the difficulty of ridding the body of absorbed radioisotopes and thus the exposure period may extend over the remaining life-span of the individual. Particularly dangerous are those alpha and beta-emitting radioisotopes which are known to be deposited in bone. Such alpha-emitting substances include radium and plutonium. The only data available for most radionuclides are derived from animal experiments. Often even these are lacking. There is a considerable amount of information in the case of radium, and it is necessary that as much as possible should be gathered and studied so that valid comparisons can be made.

In dial painters, a body burden of radium was acquired through oral ingestion over a period of several years. After a varying delay period, diseases of bones and joints resulted from this. There are three groups of investigators in the U.S.A. and several other groups in other countries who are making a systematic study of individuals who have acquired a body burden of radium. The study covers not only those whose acquisition of radioactive substances was through occupational exposure, like the dial painters; but also patients who were given radium salts as a medicament either orally or parenterally for many non-malignant conditions by physicians in the period $1910-1930$.

After intake, excretion of radium is incomplete and build-up occurs in the skeleton with deposition in those osteons that are being formed at that time giving "hot spots." Subsequent bone turnover leads to diffusion of part of the deposit. If the radium concentration is high enough at any site, the bone is, for the most part, dead bone by twenty to forty years after deposition at that site. There is continuous irradiation of all cells embedded in the bone and many cells of the periosteum and endosteum from the alpha and beta particles and X-rays produced by the radioactive disintegration of radium through its decay chain to lead 206 (radium G).

This may lead to tumous of mesenchymal cells (sarcomata) and of epithelial cells (carcinomata) in immediate apposition around the bone. The sarcomata may or may not form new bone, and most frequently resemble fibrosarcomata or spindle-cell sarcomata, and typically, metastasize widely. Carcinomata arise in the epithelium lining the mastoid air cells, the paranasal sinuses and in the mouth especially in the alveolar ridge of the maxilla. The lesions encountered in radium-bearing patients include aseptic necrosis of the head of the humerus, femur, radius and tarsal scaphoid, and pathological fractures are seen in advanced cases, affecting most frequently the femur, humerus and patella. Such fractures occur in the absence of trauma or after trivial stress.

The radiographic changes in bone and joints have been described by many authors and a pattern, characteristic of these patients, can be seen although the individual findings of the pattern are not unique, and resemble those related to vascular changes in bone. The earliest changes are small areas of bone resorption in the cortex of the shaft of tibia, fibula, radius and ulna, and similar "punched out" areas may also be seen in the bones of the skull. Areas of dense bone sclerosis are seen in the head and neck of the femur and humerus, in the pelvis and in the region of the epiphysis in the extremities. These sclerotic lesions simulate those of caisson disease. Coarsening of the trabecular structure of the bone is seen. Aseptic necrosis of the head of the humerus, radius, femur and tarsal scaphoid occur.

Thus atypical bone necrosis, pathological fractures, osteosarcomata and unusual tumours of the head and neck occurring in late adult life is the picture seen in these patients.

It is noteworthy that some radio-active materials are unique amongst deleterious chemicals in that their presence in the body can be detected and measured by physical apparatus external to the body. Accurate estimates of the body content of radium can be made by gamma-ray spectroscopy with large scintillation crystals and analysis of theo expired breath for radon. These data, with X-ray studies of the entire skeleton and haematological studies, are used in an attempt to link the lesions seen with the level of retained radium in the body. It is only in the case of radium that there is any considerable amount of information and in all other cases of bone-seeking nuclides the prediction of permissible levels of body radioactivity has been done by a comparison of the energies per disintegration emitted by the radionuclide and radium. For all other radionuclides, experience of the effects of chronic whole-body irradiation from external sources of $\mathrm{X}$ - and gamma-radiation is applied and the assumption is made that external radiation dose levels are equally applicable to irradiation from radioactive body burdens. This is necessary because for most radionuclides the only data are from animal experiments and often these are lacking. The most comprehensive account is in the Report of Committee II of the International Commission on Radiological Protection (I.C.R.P. II). This report recommends values for the maximum permissible body burden (M.P.B.B.) of most of the radionuclides available at present.

In a study of 300 individuals bearing a significant body content of $226 \mathrm{Ra}$, no bone tumours have been found where the total body burden was below $0.4 \mu c$.

As far as operational control of those occupation- 
ally exposed to radionuclides is concerned, it is necessary to be able to detect significant amounts of radioactive materials within the body in order to be able to advise whether further intake should be reduced or prevented. In some cases the routine monitoring of occupationally exposed persons may be advisable. Many of the radionuclides listed in I.C.R.P. II can be detected with quite simple apparatus at levels below those considered acceptable. If the isotopes emit gamma rays of $100 \mathrm{keV}$ or more, the probability of detection is so high in these cases as to make the method promising for protective control methods and in hospital work. In some cases more sophisticated apparatus is necessary and more elaborate methods and it is necessary to have a shielded room.

These direct methods must be supplemented by indirect methods such as assay of excreta. Routine excretion analysis is also needed to supplement methods of environmental contamination survey of radioactivity, to detect unsuspected intakes of radioactive material and hence to evaluate the desirability or necessity of follow-up and future management of the case. Every case of significant exposure merits careful individual examination. Individual variation in renal clearance is very wide in normally healthy human subjects, faecal excretion poses its own problems; and the precision with which evaluation of retention can be made from bioassay data is limited. The WHO/FAO Report on Methods of Radiochemical Analysis states "Estimation of body burden continues to be one of the most difficult problems in radiological protection." Despite all the difficulties, the assay of excreta, when applied intelligently to a specific problem, gives most valuable information for radiation control. It is very desirable that all the circumstances of the case should be put together and the results from environmental monitoring assessed in conjunction with the results from personal monitoring by external counting and sample assays of excreta to reach as accurate a diagnosis as possible.

The very high degree of protection afforded the workers in the Atomic Energy industry has been so effective that no cases of bone pathology or bone tumour have been seen that can be causally related to plutonium or radioactive strontium in the skeleton, and have not posed a practical problem.

The practical aspects and the academic scientific inquiry into the mechanism of carcinogenesis have become closely interwoven in a discussion of the concept of "threshold" under the pressure of industrial and political situations in recent years. In experimental studies, set up to correlate the probability of tumour development with radiation dose, a large variety of dose-effect relationships appear. Differing experimental conditions - the species of animal used, the animal strain, the tumour observed
- produce different relationships. Much of the present discussion with regard to the presence ob absence of a threshold concerns the induction of. leukaemia. The International Commission on Radiological Protection notes that "it may be postulated that if the dose is lower than a certain threshold value no leukaemia is produced ....... 음 In this case it would be necessary to estimate the threshold dose and make allowance for recovery? There is not sufficient evidence to do this, but cautiont would suggest that an accumulated dose of 758 rems might exceed the threshold."

Retrospective surveys of the incidence of leu $\vec{\omega}$ kaemia in radiologists, in patients treated bog radiotherapy, particularly cases of ankylosing spondylitis and in irradiated Hiroshima bomb. survivors, provide the evidence for radiation $\hat{+}$ induced leukaemia in man. Surveys of a population of exposed and non-exposed persons and the re? corded leukaemia incidence in the two groups haver shown increased incidence after irradiation. In cases of spondylitis treated by radiation, it was shown that external irradiation of part of the body including an appreciable portion of the bone? marrow with doses of 500 rads or more, produce of an increased incidence of leukaemia in man.

The Japanese survivors showed a progress $8 / \overrightarrow{\mathrm{B}}$ increase in leukaemia incidence reaching a pea between the 4th and 7th post-atomic-bomb yeakis Individual dose values are open to several sourees of error and dose estimations can only be approxis mate. In the spondylitis series, there were noe enough spondylitis non-irradiated persons to forn? a control group so the general population was used as a control.

The fundamental difficulty is that the mechanism of carcinogenesis is unknown. Considerable work on mouse leukaemia has been carried out, but much. less on bovine and canine leukaemias. There are number of similar features between the solitary and diffuse lymphoid tumours with or without a leu긍 kaemic blood picture which arise in animals and human leukaemias. Some comparison is un윽 doubtedly possible although the terminology and classification schemes are not well defined, thus making it a difficult matter to compare human with? animal lesions. The difficulty which has been: encountered in the attempts to extrapolate the datß to derive a dose - response relationship has prevented a definitive answer being given to the question whether or not a "threshold dose" exists below which there will be no induction of cances? or leukaemia.

Constitutional effects, as in the acute radiation syndrome, result from a whole body radiation dose An early lethal effect, or one immediately lethal ${ }_{0}^{\circ}$ may result from a flash dose of 600 rads: the 50 peet cent lethal dose in man is thought to be in the region 
of 350 - 450 rads while the smallest dose of radiation given in a short time which produces an observable effect is about 25 rads. In the acute radiation syndrome, the haemopoietic system and gastrointestinal tract show the most severe clinical effects, and the clinical response in man to whole body radiation is related to dysfunction of the intestine and loss of the immune and antibacterial properties of the body in consequence of aplasia of the bone marrow. If damage to such essential tissue as intestinal epithelium and marrow is sufficiently severe, death can result, but in whole-body exposure of animals in the lethal dose range, surviving animals show various disabilities - they develop cataract, are sterile, underweight and die younger than controls. Most somatic effects are related to the rate at which the radiation is given because if given slowly, repair processes and recovery proceed pari passu.

There is little direct information on the genetic effects of radiation in man: only exposure of the germ cells in ovary and testis, and the total amount of radiation they receive are involved in this. Radiation increases the rate of mutation, and mutant genes in the cells of the germinal tract can be transmitted to the next and future generations. Probably there is no threshold, although even in convenient experimental material, observations are very difficult - if not quite impossible - at very low dosages.

"Mutation" is taken to include major changes in chromosome structure as well as point mutations affecting single genes. It was thought that increased mutation rate was more or less directly proportional to the amount of additional radiation dosage. Now it appears that probably the amount of mutation is increased disproportionately with increased dosage.

Estimates of possible genetic damage can only be made with a very wide margin of uncertainty. The convention is to consider "the doubling dose," that is the dose of radiation which would double the existing mutation rate assuming an exposure period from birth to 30 years of age. The United Nations Scientific Committee suggest that it is in the range of $10 \mathrm{r}$ to $100 \mathrm{r}$ : the Medical Research Council put forward $30 \mathrm{r}$ to $80 \mathrm{r}$. The peacetime exposure is only a small fraction of these amounts, but it is desirable that the dose to the gonads should be kept as low as possible: in diagnostic radiology it can be kept extremely low and in many cases avoided altogether by shielding.

There is considerable interest in the possibility of risk to children and babies from radiation and of the possibility of miscarriage and stillbirth resulting from irradiation during pregnancy. After very heavy dosage, as from a nuclear weapon explosion, there is no doubt that this can occur - otherwise, it is not a problem unless the dose of radiation is large. Experimental irradiation of pregnant animals has shown that the radiosensitivity of embryonic tissue varies considerably. The critical period is the stage of cell differentiation. In mouse experiments, the radiation hazard is greatest at the time of major organogenesis and doses as low as $25 \mathrm{r}$ are effective in producing changes and causing local deficiencies and malformations if applied at this sensitive stage. Later during the period of growth of the foetus, sensitivity drops to low levels. Thus the time-factor in the pregnancy is important and governs the types and degrees of damage from irradiation. Doses high enough to produce abnormalities at the critical early period do not necessarily cause abortion or pre-natal death. It has been suggested that in women of child-bearing age, diagnostic irradiation of the uterus should, if possible, be carried out in the two weeks following the last menstrual period even if the dosage is less than $25 \mathrm{r}$.

In preventive measures, the progress made has kept pace with the speed of the advances in nuclear technology in harnessing nuclear energy for power for use as electricity and for marine propulsion. Possible credible accidents to reactors have been assessed to see to it that an appreciable hazard to surrounding populations will not be produced. Some reactors are enclosed in outer shells, so that any large-scale leakages of radioactivity resulting from an accident are contained inside and do not escape to the atmosphere.

In radiation protective practice, the association and co-operation of people with diverse training and experience including engineers, chemists, physicists and medical practitioners has achieved an unique safety standard. The international co-operation in formulating recommendations for radiological protection has also been unique and has resulted in a singularly uniform standard. There is such clearly defined guidance on standards of shielding and permissible radiation levels, supported by such detailed codes of practice that the health physicist is enabled to maintain a very efficient technical control of radiation in industrial and hospital practice. Medical supervision is based essentially on the principles of sound occupational practice and in industry, medical advice on job placement is directed towards the specific work assignment rather than radiation hazards. Small groups working in relative isolation or the individual working alone stand in most need of care, advice and education. In future, the aim should be to train the workers to understand the principles of radiation protection and to assume more responsibility in carrying out the procedures necessary for environmental control. In the era which lies ahead, the exploitation of the vast resources placed at the disposal of mankind in the development of nuclear energy for power and in the applications of radioactive materials can undoubtedly be carried out as safely as in the past twenty years of development on the basis of protective measures which have now been laid down. 